\title{
Implications of controlled short-wavelength light exposure for sleep in older adults
}

\author{
Mariana G Figueiro*, Natalia Z Lesniak and Mark S Rea
}

\begin{abstract}
Background: Environmental and physiological conditions make older adults more likely to lose synchronization to their local time and experience sleep disturbances. A regular, 24-hour light/dark cycle promotes synchronization. It is now well established that the circadian system is maximally sensitive to short-wavelength (blue) light. The purpose of the present study was to measure dose effectiveness (amounts and durations) of short-wavelength (blue) light for stimulating the circadian systems of older adults. We investigated the impact of six corneal irradiances $\left(0.7\right.$ to $\left.72 \mu \mathrm{W} / \mathrm{cm}^{2}\right)$ of $470-\mathrm{nm}$ light on nocturnal melatonin production. Nine participants, each over 50 years of age completed a within-subjects study. Each week, participants were exposed to one of the six irradiances of 470-nm light for 90 minutes.
\end{abstract}

Findings: A two-factor ( 6 corneal irradiances $\times 10$ exposure durations), within-subjects analysis of variance (ANOVA) was conducted using the melatonin suppression levels. The ANOVA revealed a significant main effect of corneal irradiance $\left(F_{5,30}=9.131, p<0.0001\right)$, a significant main effect of exposure duration $\left(F_{9,54}=5.731, p<\right.$ 0.0001), and a significant interaction between these two variables $\left(F_{45,270}=1.927, p<0.001\right)$. Post hoc $t$-tests revealed that corneal irradiances as low as $2 \mu \mathrm{W} / \mathrm{cm}^{2}$ reliably suppressed melatonin after 90-minute exposure whereas $0.7 \mu \mathrm{W} / \mathrm{cm}^{2}$ did not.

Conclusions: Sleep disorders are common and a serious problem for millions of older adults. The present results showed that comfortable, precise and effective doses of light can be prescribed to older adults to reliably stimulate the circadian system that presumably would promote entrainment and, thus, regular sleep. Field studies on the impact of short-wavelength-light doses on sleep efficiency in older adults should be performed.

\section{Introduction}

A large and growing body of research shows that a regular, 24-hour light/dark cycle synchronizes circadian rhythms in all species, including humans, to local time on earth. Light levels required to activate the circadian system are higher than those required for vision [1]. Since older adults are less active and tend to stay indoors more often where light levels tend to be low, they may not be exposed to lighting that sufficiently stimulates their circadian system [2]. Moreover, these same older adults commonly have less light reaching their retina due to natural age-related reductions in pupil size (senile miosis) and in crystalline lens transmission [3]. Together, these environmental and physiological conditions make older adults more likely to lose entrainment to their local

\footnotetext{
* Correspondence: figuem@rpi.edu

Lighting Research Center, Rensselaer Polytechnic Institute, 21 Union Street/
} $3^{\text {rd }}$ floor, Troy, NY, 12180, USA time and, as a result, more likely to experience sleep disturbances $[2,4,5]$.

Timed light treatments have been shown to improve circadian entrainment in older persons, including those with Alzheimer's disease (AD), a population that is perhaps most susceptible to circadian disruption through light restriction [6]. Clinical research has shown, for example, that exposing persons with AD to light levels higher than what they would normally experience in their homes or in institutions during the day and darkness at night can consolidate their rest/activity patterns $[7,8]$. Morning and evening bright light exposures ( $>2,500$ lux at the cornea) have been shown to improve nighttime sleep, increase daytime wakefulness, reduce evening agitation behavior, and consolidate rest/activity patterns of persons with $\mathrm{AD}$ [8-11]. Riemersma-van der Lek and colleagues demonstrated that all-day exposure to high levels of a white light (i.e., at least 1000 lux at the eye of a $4100 \mathrm{~K}$ light source)
C Biomed Central

() 2011 Figueiro et al; licensee BioMed Central Ltd. This is an open access article distributed under the terms of the Creative Commons Attribution License (http://creativecommons.org/licenses/by/2.0), which permits unrestricted use, distribution, and reproduction in any medium, provided the original work is properly cited. 
improved sleep efficiency and cognition in patients with $\mathrm{AD}$ as well as reduced symptoms of depression [12]. Light applied in the evening has also been shown to be effective in phase delaying the circadian system of older people without dementia, allowing them to fall asleep later and wake up later [13].

Although bright light interventions appear to be effective for promoting circadian entrainment and improving sleep efficiency, they can be expensive, time consuming for caregivers, and uncomfortable for older adults, who show symptoms of photophobia. In fact, there is evidence that light treatment may not always result in better sleep in older adults. Light treatment alone (1 hour of 2,500 lux at the angle of gaze) did not improve nighttime sleep, daytime wake and rest activity in AD patients $[14,15]$. These negative results may reflect problems with compliance, so there is a need to develop more practical and, thus, more effective methods for light delivery.

Treatment efficacy (i.e., effectiveness per electrical watt) is also important to consider for practical applications. One simple method for improving treatment efficacy is placing the light source closer to the eyes. The irradiance at the cornea falls off with the square of the distance from the source, so small reductions in this distance have disproportionately large increases in light exposure to the retina. Tuning the light spectrum for maximum effect also increases treatment efficacy. Research has demonstrated that the spectral sensitivity of the circadian system differs from the spectral sensitivity of the achromatic visual channel, which is used for such visual activities as reading. Short-wavelength (blue) light between approximately $440 \mathrm{~nm}$ and $470 \mathrm{~nm}$ is maximally effective at stimulating the human circadian system, as measured through acute melatonin suppression and phase shifting of the melatonin rhythm [16-18].

One way to provide older adults with inexpensive, lowpowered light sources that provide sufficient levels of circadian-effective light would be to mount short-wavelength light-emitting diodes (LEDs) on spectacle frames. With a thoughtful design, the intensity distribution of the circadian-effective light sources should not interfere with the performance of visual tasks. Some engineering sophistication is also needed to precisely control the timing and duration of the light dose. Temporally controlled 470-nm (blue) LEDs mounted on spectacle frames would be inexpensive, require little, if any, maintenance, and would be comfortable and safe for users as long as the dose was properly prescribed. A prototype of the device was developed and its effectiveness at two irradiances was measured previously by Figueiro et al [19].

The purpose of the present study was to extend the findings from Figueiro et al [19] to more precisely measure dose effectiveness (amounts and durations) of shortwavelength (blue) light for stimulating the circadian systems of older adults. A self-powered, blue-light goggle system had been developed for the previous study and was used again here to deliver the light doses. Nocturnal melatonin suppression was used as the measure of the threshold for light activation and of dose efficacy. In addition, these data would be expected to be used to refine the mathematical model of human circadian phototransduction [17] to predict circadian effectiveness for other light sources. Once the dose effectiveness for short-wavelength stimulus is known, the dose effectiveness for other narrowband sources can be estimated from the modeled spectral sensitivity function.

\section{Methods}

The prototype blue-light goggles were developed under a grant from the National Institutes on Aging to deliver circadian-effective light doses to adults 50 years of age and older. Previously, nocturnal melatonin suppression was measured at just two light doses from these blue-light goggles [19]. The present study was designed to extend those findings by measuring the effectiveness of additional doses from these goggles for suppressing nocturnal melatonin in older adults. The Institute Review Board (IRB) of Rensselaer Polytechnic Institute approved the present experimental protocol and all subjects were asked to sign a written informed consent form.

\section{Subjects}

Of the eleven subjects who completed the previous study [19], nine were still available for further study; therefore only data for the nine subjects who completed all experimental conditions are included here. These subjects had been previously recruited through e-mail notices, posters, and word-of-mouth. Eligibility for the study required subjects to be over the age of 50 years and to be free of any major health problems, such as cardiovascular disease, diabetes, or high blood pressure. Potential subjects were excluded if they were taking over-the-counter melatonin or prescription medication such as blood pressure medicine, antidepressants, sleep medicine, hormone replacement therapy or beta-blockers. Potential subjects who stated they had any eye disease (such as cataract, glaucoma and color blindness) were not accepted into the study. Seven of nine subjects completed the Munich ChronoType questionnaire (MCTQ) [20]. Because the experiment would be conducted in the early part of the night, extreme late chronotypes were excluded to assure melatonin levels would be high at the time of the experiment; of the seven subjects who completed the MCTQ, all were moderate to early chronotypes with a mean \pm standard deviation of $1.3 \pm 1.0$. All subjects contributing to the present results were between 51 and 62 years of age (mean \pm standard deviation, $56.2 \pm 3.9$ years). 


\section{Protocol}

Subjects who completed the 2-night protocol reported by Figueiro et al [19] took part in the additional five-night protocol; the sessions took place on Friday nights (between September 2009 and April 2010) and were scheduled at least one week apart. On the weeks of the experimental sessions, subjects were instructed to go to bed between 21:00 and 23:00. On the day of the experiment, subjects were asked to refrain from caffeine and alcohol 12 hours prior to the start of the experiment and to arrive at the lab at 22:00. Upon arrival, a registered nurse inserted an indwelling catheter into an arm of each subject. At 23:00, room lights were turned off, except for two red LED traffic signal lights $\left(\lambda_{\max }=640 \mathrm{~nm}\right)$ placed on the floor, outside the subjects' field of view. These traffic signals provided indirect illumination in the space, less than 1 lux at the cornea, that was sufficient for people to safely navigate in the space without stimulating their circadian systems.

After the room lights were turned off, the subjects were seated and watched a movie that was projected on a screen about 20 feet ( 6 meters) away. The measured (polychromatic) illuminance at the subjects' cornea reflected from the projection screen alone ranged from 0.14 to 0.2 lux; this level is too low to stimulate the circadian system [21]. The movie kept subjects awake, minimizing the likelihood of eye closures. Moreover, experimenters continuously monitored the subjects to ensure that they had their eyes open. Subjects were not allowed to drink or eat after 23:30.

Table 1 shows the times that blood and saliva samples were taken from the first subject in a session. To allow the nurse to collect the blood samples from every subject in a session, data collection times for the other subjects were each staggered by about 2 minutes. The first two blood and saliva samples were collected at the same time (23:50 and 00:00). Immediately after the second sample was collected from the subjects, they were asked to put on the blue-light goggles (described below), which remained on for the remainder of the experimental session.

One saliva and three blood samples were collected from each subject at each of seven appointed times in a 90-minute session. Saliva samples were obtained using the Salivette system (ALPCO Diagnostics, Salem, NH, USA). Three, 3 -ml blood samples were drawn by the nurse at each of the prescribed times; the first sample was always discarded and the second and third samples were spun in a centrifuge for 15 minutes. Frozen saliva and plasma samples were subsequently sent to an independent laboratory (Pharmasan Labs, Osceola, WI) for melatonin radioimmunoassay. The sensitivity of the saliva assay was $0.7 \mathrm{pg} / \mathrm{ml}$ and the intra- and inter-assay coefficients of variability (CVs) were $12.1 \%$ and $13.2 \%$, respectively. The sensitivity of the plasma assay was $3.5 \mathrm{pg} / \mathrm{ml}$ and the intra- and inter-assay CVs were $8.1 \%$ and $14.8 \%$, respectively.

All subjects saw all lighting conditions described in Table 2 in a counterbalanced order within a set of sessions. Thus, different light exposures were provided to subjects on any given night. When experiencing the dark (control) condition, subjects wore non-energized bluelight goggles. Melatonin concentrations in saliva and in plasma were assayed at different times as three different batches, also shown in Table 2 .

At completion of pupil measurements (described below), room lighting was turned on and the registered nurse removed the indwelling catheter from the subjects' arms and subjects were offered a ride home.

\section{Light Levels}

Topbulb.com produced the blue-light goggles used to deliver the prescribed doses of light from LUXEON Rebel (LXML - PB01) LEDs. Subjects wore the goggles while they received one of seven irradiance levels at the cornea, including the dark control condition without energizing the LEDs. Table 2 shows the six average irradiance $\left(\mu \mathrm{W} / \mathrm{cm}^{2}\right)$ and illuminance (lux) levels, as well as the peak and full width at half maximum (FWHM) wavelengths generated by the energized goggles.

Four LEDs, two per goggle lens, with peak wavelengths ranging from 466 to $474 \mathrm{~nm}$ were mounted to the top inside rim of the goggles. The LEDS were located behind a translucent plastic tube to diffuse the light and to reduce point-source glare [22]. Although the amount of light emitted by the LEDs was below levels that might be of concern for blue light hazard [23], diffusing the light sources further ensured safety. Heat from the LEDs was dissipated via a heat sink strip. The LEDs were connected by wire to a small control case $(0.5 \times 1.5 \times 2.5$ in. $[1.3 \times$ $3.8 \times 6.4 \mathrm{~cm}])$, which held a 3.0 VDC two-battery power source, and the control circuitry, including a Texas Instruments voltage regulator (TPS77018DBVR $1.8 \mathrm{~V}$ ). The small control case also housed the LED driver (Maxim Evaluation Kit -1848 EVKIT), which was used to

Table 1 Data collection times for the first subject in a session to provide blood and saliva samples

\begin{tabular}{|c|c|c|c|c|c|c|c|}
\hline & Dark & Dark & Goggles on & Goggles on & Goggles on & Goggles on & Goggles on \\
\hline Blood & 23:50 & 00:00 & 00:10 & $00: 20$ & 00:30 & 01:00 & 01:30 \\
\hline Saliva & 23:50 & 00:00 & 00:05 & 00:15 & $00: 25$ & $00: 45$ & $01: 15$ \\
\hline
\end{tabular}


Table 2 Lighting conditions presented to subjects

\begin{tabular}{llllll}
\hline Batch & $\begin{array}{l}\text { Irradiance } \\
\left(\boldsymbol{\mu} \mathbf{W} / \mathbf{c m}^{2}\right)\end{array}$ & $\begin{array}{l}\text { Illuminance } \\
(\mathbf{l u x})\end{array}$ & $\begin{array}{l}\text { Peak wavelength } \\
(\mathbf{n m})\end{array}$ & $\begin{array}{l}\text { FWHM } \\
(\mathbf{n m})\end{array}$ & $\begin{array}{l}\text { Pupil diameter } \\
(\mathbf{m m})\end{array}$ \\
\hline 3 & dark & NA & NA & NA & $5.5(5.4 \pm 0.51)$ \\
\hline 3 & $0.7(0.7 \pm 0.04)$ & $0.61(.6 \pm 0.16)$ & $473(473 \pm 0.4)$ & $18(18 \pm 0.83)$ & $5.3(5.2 \pm 0.52)$ \\
\hline 3 & $2.0(2 \pm 0.08)$ & $1.2(1.5 \pm 0.37)$ & $462(467 \pm 5.5)$ & $18(18 \pm 0.29)$ & $4.9(4.8 \pm 0.53)$ \\
\hline 1 & $6.1(6 \pm 0.2)$ & $5.8(6.8 \pm 3.1)$ & $474(474 \pm 0.3)$ & $20(20 \pm 0.3)$ & $4.2(4.4 \pm 0.61)$ \\
\hline 2 & $11(11 \pm 2.6)$ & $11(11 \pm 1.6)$ & $474(474 \pm 1.8)$ & $20(20 \pm 0.5)$ & $3.8(4.1 \pm 0.91)$ \\
\hline 1 & $20(20 \pm 0.4)$ & $12(14 \pm 4.1)$ & $462(466 \pm 5.9)$ & $19(19 \pm 1.1)$ & $3.8(4.1 \pm 0.87)$ \\
\hline
\end{tabular}

Medians and, in parentheses, means \pm standard deviations of corneal irradiance, corneal illuminance, peak wavelength and full width at half maximum (FWHM) wavelength delivered by the blue-light goggles used in the experiment. Peak wavelengths vary slightly because different goggles were used to deliver the different light levels. Medians, means \pm standard deviations of measured subject pupil diameter while wearing the blue-light goggles at the prescribed levels are also provided. Pupil areas and corneal irradiances or corneal illuminance levels were used to estimate retinal light doses. Melatonin concentrations in saliva and in plasma were assayed at different times as three different batches.

adjust the flux emitted by the LEDs to the prescribed light level in a session.

Prior to every session, new batteries were inserted into the small control case. While the LED driver was adjusted, light from the left and from the right goggle lens LEDs were alternately measured with a Model 2300i Action Research double monochrometer a Spectra Sense Version 4.3.0 spectral radiometer using a $5 \mathrm{~mm}$ diameter optical fiber ending in a lambertian diffuser as the sensor. During measurements, the sensor was placed where the subject's corneas would be while wearing the goggles. The average irradiance from the left and right lenses was used to meet the prescribed light level.

After each session, while a subject continued to wear the blue-light goggles at the session-prescribed corneal irradiance, ten digital images of one eye of each subject were acquired with a Sony video camera. To improve camera image quality, an infrared (IR) source irradiated the eye. Pupil size is unaffected by this supplemental source because the retina is insensitive to IR radiation. Subjects held a small ruler just below their eye during the image capture as a reference, linear scale to estimate pupil diameter. The captured image was printed and, using precise calipers, the experimenter measured both the size of the known ruler unit (e.g., $1 \mathrm{~cm}$ ) and the size of the unknown pupil diameter in the image. The ratio of the measured ruler unit from the digital image to the known ruler unit was then used to scale the measured pupil diameter from the digital image to estimate the actual pupil diameter. Three images were arbitrarily selected and used to estimate the pupil diameter for a given subject. Thus, the reported median pupil diameters in Table 2 are each based upon the median of 27 pupil diameter estimates (9 subjects $\times 3$ samples). From the median pupil diameters, pupil areas were calculated. Since there was no evidence of anisocoria with any subject, the size of both the left and right pupils should always be the same. Binocular retinal light exposures were defined as the product of the median corneal irradiance and the median pupil area in units of deka-nanowatts $\left(\mu \mathrm{W} / \mathrm{cm}^{2} \times \mathrm{mm}^{2}\right)$ or micro-lumens $\left(\mathrm{lm} / \mathrm{m}^{2} \times \mathrm{mm}^{2}\right)$ where corneal illuminance is considered.

\section{Data Analyses}

To more precisely determine the differential effects of light exposure level and duration on melatonin concentrations a series of normalizations were undertaken to minimize systematic differences among the nine subjects, between the two biomarker types (plasma and saliva), and among assay batches (Table 2). This approach was the same as that employed in the previous study by Figueiro and colleagues [19], except for the additional normalization for assay batches. Although the assay methodology was not changed, the absolute melatonin concentrations differed systematically among the three batches sent for melatonin assays as revealed by examination of the saliva and plasma concentrations at 23:50 and 00:00, the two times, in the dark, common to all experimental sessions. First, the raw melatonin concentrations in a batch, either plasma or saliva, were averaged, and the ratio of the batch average concentrations to the grand average concentrations were used to normalize the different batches for plasma and saliva concentrations. The same procedure was then followed to normalize differences among subjects. Thus, any systematic differences among batches, among subjects, and between plasma and saliva melatonin concentrations were minimized. The subject's normalized melatonin concentrations, both plasma and saliva, for every exposure duration at a given retinal irradiance were then fitted with a polynomial equation using a least squares criterion. Each of those resulting normalized melatonin concentration curves, one for every subject at every retinal irradiance, including dark, were then adjusted to a common melatonin concentration value at 00:00; the adjustment was based upon the reciprocal of the polynomial values at 00:00 for combined normalized plasma and saliva data. 
Melatonin suppression for each subject $\left(\mathrm{ms}_{\mathrm{n}} \%\right)$ was then calculated from the fitted melatonin concentration values at each retinal irradiance and for all exposure durations; the mean melatonin suppression ( $\mathrm{ms} \%$ ) levels shown in Figure 1 were based upon mean suppression levels from every subject at each retinal irradiance and every exposure duration. Suppression levels for a given subject were determined by the ratio of the melatonin concentrations after exposure to a given retinal irradiance for a given duration after 00:00 to the melatonin concentrations during the dark night at the same clock time.

\section{Results}

\section{Melatonin Suppression}

Figure 1 shows mean nocturnal melatonin suppression levels following exposure to each retinal irradiance level at every exposure duration for both the plasma and the saliva samples. A two-factor ( 6 corneal irradiances $\times 10$ exposure durations), within-subjects analysis of variance (ANOVA) was conducted using the melatonin suppression levels (SPSS version 13). The ANOVA revealed a significant main effect of corneal irradiance $\left(\mathrm{F}_{5}, 30=\right.$ 9.131, $\mathrm{p}<0.0001)$, a significant main effect of exposure duration $\left(\mathrm{F}_{9,54}=5.731, \mathrm{p}<0.0001\right)$, and a significant interaction between these two variables $\left(\mathrm{F}_{45,270}=1.927\right.$, $\mathrm{p}<0.001)$. Table 3 shows the results of 60 post hoc, twotailed one sample $\mathrm{t}$-tests to determine if the suppression levels were significantly different than zero. Entries in the table are the independent probabilities of a Type 1 error. The Bonferonni-adjusted criterion probability of a Type 1 error is 0.0008 ; probabilities less than this criterion are marked with asterisks in Table 3. The tendency for significant post hoc comparisons to lie in the bottom right corner of Table 3 illustrates the significant interaction between exposure duration and corneal irradiance.

In order to estimate threshold values for melatonin suppression by the 470-nm light, we compared the melatonin concentrations in the dark to those after exposure to $0.7 \mu \mathrm{W} / \mathrm{cm}^{2}$ and $2 \mu \mathrm{W} / \mathrm{cm}^{2}$. Figure 2 shows a plot of the mean differences between the measured plasma melatonin concentrations in the dark and those at both 0.7 $\mu \mathrm{W} / \mathrm{cm}^{2}$ and $2 \mu \mathrm{W} / \mathrm{cm}^{2}$ at the same exposure durations. These data were used because melatonin assays for these three corneal irradiance conditions were performed as a single batch. Post hoc 2-tailed t-tests were performed to verify if the difference values were reliably different than zero. The melatonin concentration differences between dark and $2 \mu \mathrm{W} / \mathrm{cm}^{2}$ were only significantly different than zero at the 90-minute exposure duration $(\mathrm{p}=0.05)$, although the differences at the 60-minute exposure duration were almost statistically reliable $(p=0.09)$. The melatonin concentration differences between dark and 0.7 $\mu \mathrm{W} / \mathrm{cm}^{2}$ were not significantly different than zero at any exposure duration, indicating that $0.7 \mu \mathrm{W} / \mathrm{cm}^{2}$ of 470

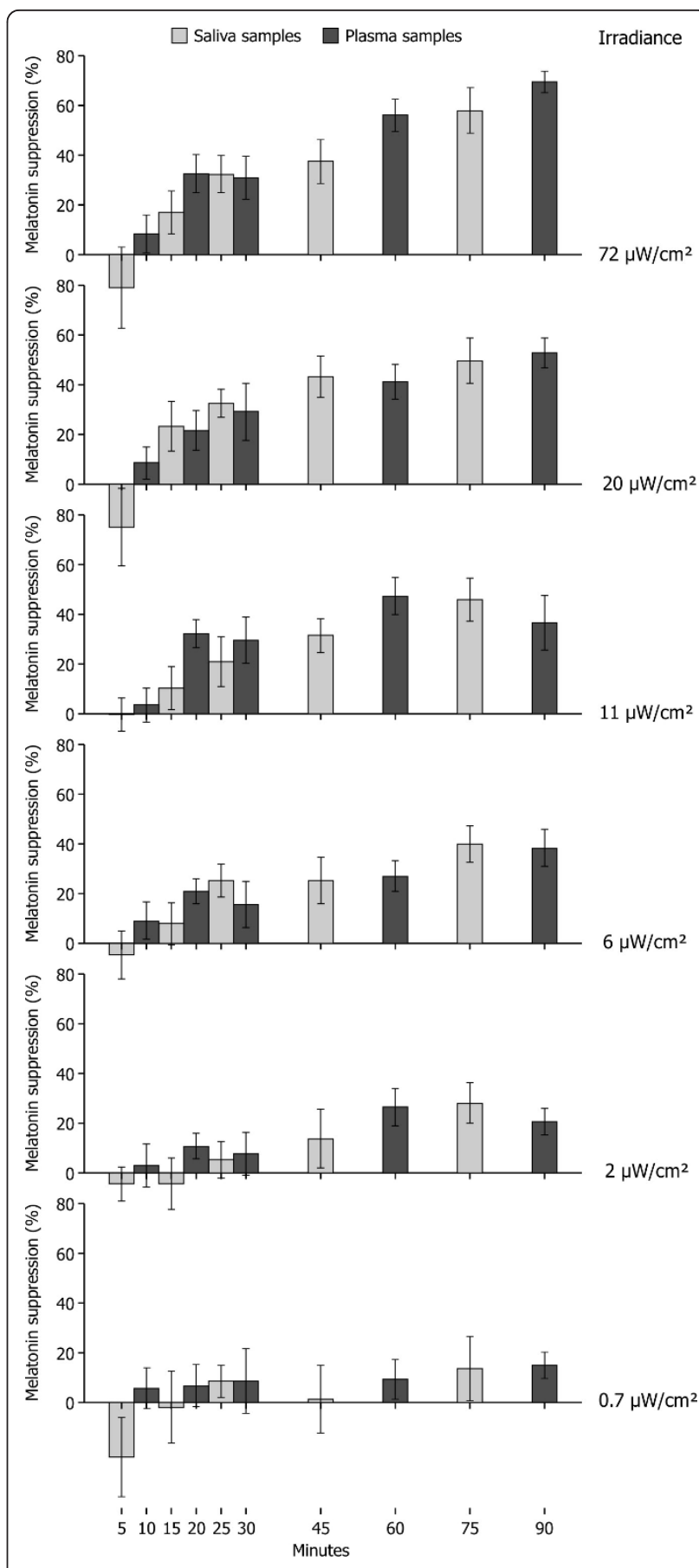

Figure 1 Melatonin suppression for saliva and plasma samples.

$\mathrm{nm}$ is very close to or at threshold for nocturnal melatonin suppression for exposure durations up to and including 90 minutes (Figure 2).

\section{Discussion}

The present results investigated the impact of 90-minute exposures from six corneal irradiances $\left(0.7\right.$ to $\left.72 \mu \mathrm{W} / \mathrm{cm}^{2}\right)$ of 470-nm (blue) light on acute nocturnal melatonin 
Table 3 Probabilities of a Type 1 error using one sample Student's t-test

\begin{tabular}{|c|c|c|c|c|c|c|c|}
\hline \multirow[b]{2}{*}{ Sample type } & \multirow[b]{2}{*}{$\begin{array}{l}\text { Exposure duration } \\
\text { (min) }\end{array}$} & \multicolumn{6}{|c|}{ Corneal irradiance $\left(\mu \mathrm{W} / \mathrm{cm}^{2}\right)$} \\
\hline & & 0.7 & 2 & 6 & 11 & 20 & 72 \\
\hline saliva & 5 & 0.210 & 0.523 & 0.634 & 0.955 & 0.303 & 0.442 \\
\hline plasma & 10 & 0.504 & 0.744 & 0.266 & 0.630 & 0.226 & 0.306 \\
\hline saliva & 15 & 0.896 & 0.688 & 0.386 & 0.273 & 0.0520 & 0.0950 \\
\hline plasma & 20 & 0.455 & 0.0768 & 0.00292 & $0.00048^{*}$ & 0.0259 & 0.0030 \\
\hline saliva & 25 & 0.231 & 0.504 & 0.00666 & 0.0725 & $0.0008^{*}$ & 0.0033 \\
\hline plasma & 30 & 0.533 & 0.414 & 0.133 & 0.0134 & 0.0353 & 0.0075 \\
\hline saliva & 45 & 0.935 & 0.284 & 0.0308 & 0.00262 & 0.0021 & 0.0039 \\
\hline plasma & 60 & 0.280 & 0.0078 & 0.00232 & $0.00021^{*}$ & $0.0004^{*}$ & $0.0000^{*}$ \\
\hline saliva & 75 & 0.329 & 0.0110 & 0.0009 & 0.0011 & 0.0009 & $0.0004^{*}$ \\
\hline plasma & 90 & 0.0246 & 0.0042 & 0.0009 & 0.0109 & $0.0000^{*}$ & $0.0000^{*}$ \\
\hline
\end{tabular}

*- statistically different than zero suppression using the Bonferroni correction for multiple t-tests.

suppression. In general, as irradiances and duration of exposure increased, melatonin suppression increased. The present data also showed that statistically significant melatonin suppression was observed after 90-minute exposure to $2 \mu \mathrm{W} / \mathrm{cm}^{2}$, suggesting that the threshold for acute suppression by a $470-\mathrm{nm}$ light is between $0.7 \mu \mathrm{W} / \mathrm{cm}^{2}$ and $2 \mu \mathrm{W} / \mathrm{cm}^{2}$ for this population.

Sleep disorders are common and a serious problem for millions of older adults [24-26]. Family members, friends, and health care workers who provide care to these individuals are also severely impacted by sleep disorders. Pharmaceuticals are commonly used to treat sleep problems with mixed success and at a fairly high cost. Light therapy to correct circadian disruption may be a practical, inexpensive, and an effective treatment alternative for older adults with sleep disorders. The present data, together with a model of human circadian phototransduction [17], enables researchers to make refined quantitative predictions of light doses and clinicians to more precisely prescribe light doses to treat circadian sleep disorders in older adults.

Administering light through a personal device does not require the person to be seated in front of a light box for a long period of time. Individuals can wear the device while performing routine tasks in the home. As shown here, although no formal collection protocol was used,

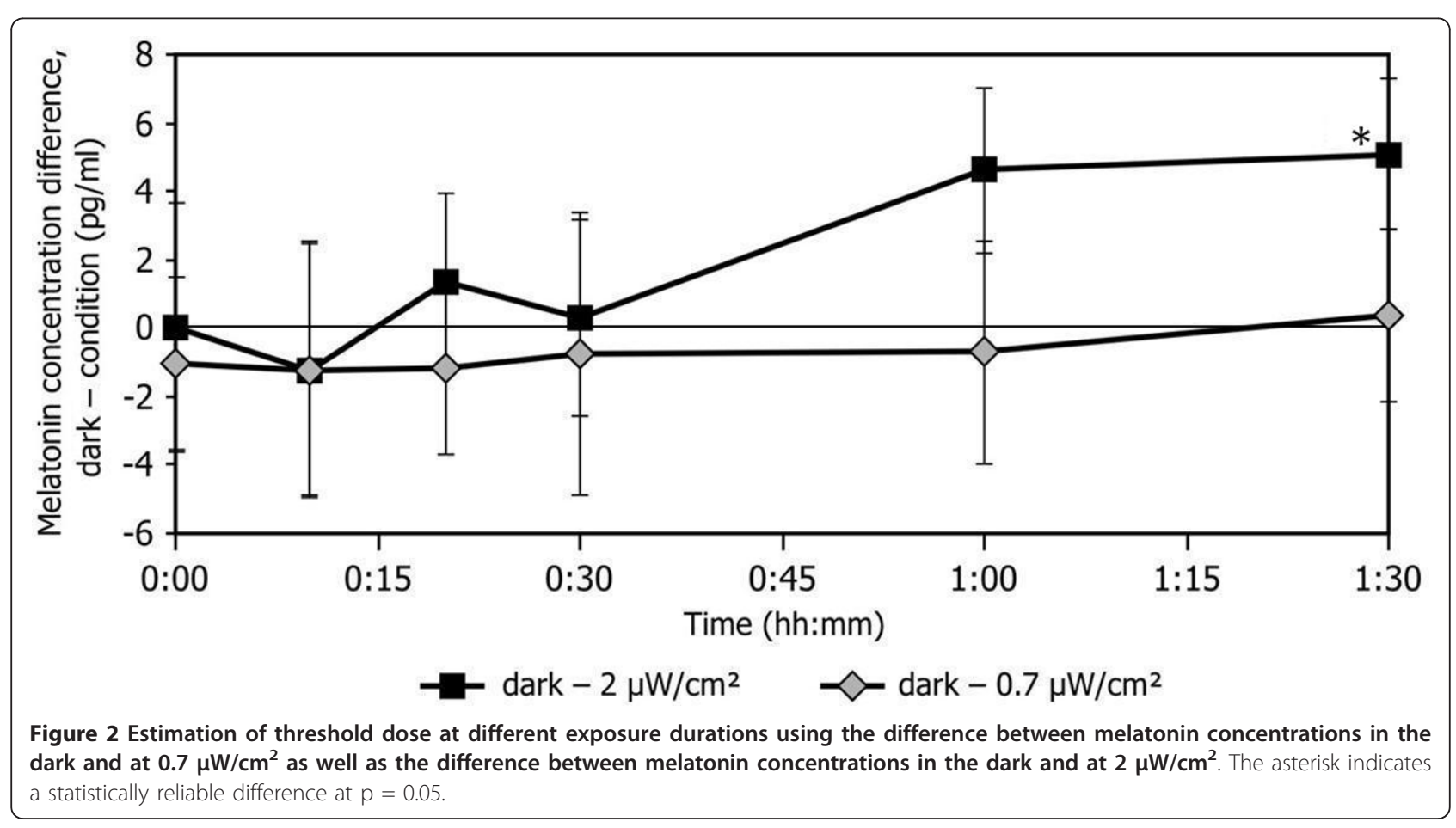


informal discussions with subjects at the end of the experiment revealed that they could comfortably watch a movie in a darkened room while receiving their light dose. As a result of tuning the temporal-spatial-spectralabsolute characteristics of light to the human circadian system, it is possible to prescribe comfortable and effective doses of light to reliably stimulate the circadian system. This tuning reduces glare and discomfort associated with bright white light and should thereby increase light treatment compliance. Simple control technologies, yet to be developed, would ensure that the light delivery is provided at the right time and for the shortest duration to promote circadian entrainment. The personal light treatment device is inherently inexpensive to fabricate, calibrate, and obviates replacing or retrofitting existing light fixtures to deliver an effective dose of light. This is especially important for many assisted living facilities or nursing homes with low budgets.

In summary, the present study was able to quantify the efficacy of a personal light treatment device for stimulating the circadian systems of older adults without risk of retinal damage [23]. In fact, the radiances utilized in the study were well below levels that pose a blue-light hazard risk [23]. Although the present data were collected in the middle of the night, this device should be used to deliver light treatment during the daytime or during the early evening hours. Additional studies are recommended to investigate the ability of short-wavelength (blue) light applied during the morning or evening hours to phase shift or to entrain the circadian systems of older adults suffering from circadian sleep disorders. Additional studies are also recommended to investigate the impact of blue-light treatment for subjective daytime sleepiness and nighttime sleep quality as well as for wake and sleep electroencephalogram. Notwithstanding these important limitations of the present study, the primary envisioned benefit of a personal light treatment device is that clinicians can begin to prescribe a precise dose of short-wavelength (blue) light for older adults to reliably stimulate their circadian systems [27]. Once a dose is determined, its brightness would be the lowest possible because the spectrum is tuned to maximum circadian effectiveness. This also has the benefit of limiting wasted heat from the LED, both improving energy efficiency and minimizing tactile discomfort. A low-brightness, battery-operated device that is safe, comfortable, and portable can provide older adults with an effective light dose while they perform their daily activities, eliminating the need to sit in front of a light box.

Two further limitations of the present study are that user acceptance was not formally evaluated and the effectiveness of blue light exposures was not tested in older adults who exhibit health problems. Although the efficacy of blue-light treatment for improving sleep efficiency in older adults, including those with $\mathrm{AD}$, has been demonstrated in earlier studies $[27,28]$, there are no empirical data to directly support acceptance, compliance, or efficacy for treatment of sleep disorders in larger clinical trials using older adults who are not as healthy as those who participated in the present study. Further, the device used in the present study is not yet a commercial product so customer feedback in other areas, like cost, was not obtained. Still, the device seems quite promising for formal tests of user acceptance.

\section{Acknowledgements and Funding}

The studies were supported in part by the Engineering Research Centers Program of the National Science Foundation under NSF Cooperative Agreement No. EEC-0812056, in part by New York State under NYSTAR contract C090145, and in part by the National Institute on Aging at the National Institutes of Health (grant number R41 AG019693). The authors would like to acknowledge Phil Bonello of Topbulb.com for providing the goggles prototype (through the NIA grant) and A. Bierman, J. Bullough, B. Erdener, K. Kubarek, B. Plitnick, B. Wood, L. Radetsky, and D. Guyon of the Lighting Research Center for their assistance with the studies and data analyses.

\section{Authors' contributions}

MGF participated in the design of the experiment, data collection, data analyses and drafted the manuscript. MSR participated in the design of the experiment, data analyses and manuscript writing. NZL participated in data collection and analyses and contributed to manuscript writing and review. All authors read and approved the final manuscript.

\section{Competing interests}

The authors declare that they have no competing interests.

Received: 6 April 2011 Accepted: 8 September 2011

Published: 8 September 2011

\section{References}

1. Rea MS, Figueiro MG, Bullough JD: Circadian photobiology: An emerging framework for lighting practice and research. Light Res Technol 2002, 34:177-190.

2. Van Someren EJ: Circadian rhythms and sleep in human aging. Chronobiol Int 2000, 17:233-243.

3. Weale R: The Senescence of Human Vision Oxford: University Press; 1992.

4. Ancoli-lsrael S, Kripke DF: Prevalent sleep problems in the aged. Biofeedback Self Regul 1991, 16:349-359.

5. Pollak CP, Perlick D: Sleep problems and institutionalization of the elderly. J Geriatr Psychiatry Neurol 1991, 4:204-210.

6. Van Someren EJ, Hagebeuk E, Lijzenga C, Scheltens P, de Rooij S, Jonker C, et al: Circadian rest-activity rhythm disturbances in Alzheimer's disease. Biol Psychiatry 1996, 40:259-270.

7. Ancoli-Israel S, Gehrman P, Martin JL, Shochat T, Marler M, Corey-Bloom J, et al: Increased light exposure consolidates sleep and strengthens circadian rhythms in severe Alzheimer's disease patients. Behav Sleep Med 2003, 1:22-36

8. Van Someren EJ, Kessler A, Mirmirann M, Swaab D: Indirect bright light improves circadian rest-activity rhythm disturbances in demented patients. Biol Psychiatry 1997, 41:955-963.

9. Mishima K, Hishikawa Y, Okawa M: Randomized, dim light controlled, crossover test of morning bright light therapy for rest-activity rhythm disorders in patients with vascular dementia and dementia of Alzheimer's type. Chronobiol Int 1998, 15:647-654.

10. Mishima K, Okawa M, Hishikawa Y, Hozumi S, Hori H, Takahashi K: Morning bright light therapy for sleep and behavior disorders in elderly patients with dementia. Acta Psychiatr Scand 1994, 87:1-7.

11. Koyama E, Matsubara H, Nakano T: Bright light treatment for sleep-wake disturbances in aged individuals with dementia. Psychiatry Clin Neurosci 1999, 53:227-229.

12. Riemersma-van der Lek RF, Swaab DF, Twisk J, Hol EM, Hoogendijk WJ, Van Someren EJ: Effect of bright light and melatonin on cognitive and 
noncognitive function in elderly residents of group care facilities: a randomized controlled trial. Jama 2008, 299:2642-2655.

13. Campbell SS, Terman M, Lewy AJ, Dijk DJ, Eastman Cl, Boulos Z: Light treatment for sleep disorders: consensus report. V. Age-related disturbances. J Biol Rhythms 1995, 10:151-154.

14. Dowling GA, Burr RL, Van Someren EJ, Hubbard EM, Luxenberg JS, Mastick J, et al: Melatonin and bright-light treatment for rest-activity disruption in institutionalized patients with Alzheimer's disease. J Am Geriatr Soc 2008, 56:239-246.

15. Dowling GA, Hubbard EM, Mastick J, Luxenberg JS, Burr RL, Van Someren EJ: Effect of morning bright light treatment for rest-activity disruption in institutionalized patients with severe Alzheimer's disease. Int Psychogeriatr 2005, 17:221-236.

16. Brainard G, Hanifin J, Greeson J, Byrne B, Glickman G, Gerner E, et al: Action spectrum for melatonin regulation in humans: Evidence for a novel circadian photoreceptor. J Neurosci 2001, 21:6405-6412.

17. Rea MS, Figueiro MG, Bullough JD, Bierman A: A model of phototransduction by the human circadian system. Brain Res Rev 2005, 50:213-228.

18. Thapan K, Arendt J, Skene DJ: An action spectrum for melatonin suppression: evidence for a novel non-rod, non-cone photoreceptor system in humans. J Physiol 2001, 535:261-267.

19. Figueiro MG, Bierman A, Bullough JD, Rea MS: A personal light-treatment device for possibly improving sleep quality in the elderly: Dynamics of nocturnal melatonin suppression at two exposure levels. Chronobiology International 2009, 26:726-739.

20. Roenneberg T, Wirz-Justice A, Merrow M: Life between clocks: daily temporal patterns of human chronotypes. J Biol Rhythms 2003, 18:80-90.

21. Zeitzer J, Dijk D, Kronauer R, Brown E, Czeisler C: Sensitivity of the human circadian pacemaker to nocturnal light: melatonin phase resetting and suppression. J Physiol 2000, 526(Pt 3):695-702.

22. Luckiesh MH, Guth SK: Brightnesses in visual field at borderline between comfort and discomfort (BCD). Illuminating Engineering 1949, , 44: 650-670.

23. Bullough JD: The blue-light hazard: A review. Journal of the Illuminating Engineering Society 2000, 29:6-14, 141.

24. Foley DJ, Monjan AA, Brown SL, Simonsick EM, Wallace RB, et al: Sleep complaints among elderly persons: an epidemiologic study of three communities. Sleep 1995, 18:425-432

25. McCurry SM, Ancoli-Israel S: Sleep Dysfunction in Alzheimer's Disease and Other Dementias. Curr Treat Options Neurol 2003, 5:261-272.

26. Van Someren EJ: Circadian and sleep disturbances in the elderly. Exp Gerontol 2000, 35:1229-1237.

27. Figueiro MG, Rea MS, LEDs: Improving the sleep quality of older adults. Proceedings of the CIE Midterm Meeting and International Lighting Congress 12-21 May, 2005 Leon, Spain. Commission Internationale de L'Eclairage; 2005

28. Figueiro MG, Saldo E, Rea MS, Kubarek K, Cunningham J, et al: Developing Architectural Lighting Designs to Improve Sleep in Older Adults. The Open Sleep Journal 2008, 12:40-51.

doi:10.1186/1756-0500-4-334

Cite this article as: Figueiro et al.: Implications of controlled shortwavelength light exposure for sleep in older adults. BMC Research Notes 2011 4:334

\section{Submit your next manuscript to BioMed Central and take full advantage of:}

- Convenient online submission

- Thorough peer review

- No space constraints or color figure charges

- Immediate publication on acceptance

- Inclusion in PubMed, CAS, Scopus and Google Scholar

- Research which is freely available for redistribution

Submit your manuscript at www.biomedcentral.com/submit
Biomed Central 